\title{
Epidemiological modelling in infectious diseases: stages and classification
}

\author{
Özge YILMAZ ÇAĞIRGAN', Abdurrahman Anıl ÇAĞIRGAN²
}

${ }^{1}$ Poultry Diseases Diagnostic Laboratory, Institute of Bornova Veterinary Control, İzmir/TURKEY

${ }^{2}$ Department of Virology, Laboratory, Institute of Bornova Veterinary Control, Izmir/TURKEY

\author{
Key Words: \\ epidemiological modelling \\ infectious diseases \\ mathematical model \\ modelling stages \\ Received : :27.02.2020 \\ Accepted $\quad: 17.05 .2020$ \\ Published Online : 30.12 .2020 \\ Article Code : 695267 \\ Correspondence: \\ Ö. YILMAZ-CAĞIRGAN \\ (yilmaz-ozge@windowslive.com)
}

\begin{abstract}
Modelling in infectious diseases has recently been an important field due to avian influenza, swine influenza, severe acute respiratory syndrome (SARS), Middle East respiratory syndromecoronavirus (MERS-CoV), novel coronavirus (nCoV) and many other diseases. Epidemiological models are usually defined as mathematical and/or logical demonstrations of epidemiology of diseases and the related process. Concerning animal disease management, 'models' can be defined more widely in that they contain a range of statistical/mathematical tools regarding other aspects of the disease in addition to its spreading. Modelling might be useful when experimental or field studies are impossible or not practical or in retrospective analyzing of previous epidemics in order to search alternative control strategies. The aim of this study was to examine some of the modelling methods and determine what mathematical modelling meant in infectious diseases, its purpose of use, to classify the steps followed during modelling period and models used in the field of animal health.
\end{abstract}

ORCID:

Ö. YILMAZ-ÇAĞIRGAN : 0000-0002-8590-1013

AA. ÇAĞIRGAN :0000-0001-7766-3150

\section{INTRODUCTION}

When deciding on control of diseases in animal health, there are still some deficiencies in how the interaction between the environment and the host has an impact on the transmission of infection and the development of the disease. Epidemiological disease models offer a path to overcome these uncertainties by combining the information obtained from field and experimental studies with opinions of experts about the dynamics of infections and disease control (1).

Epidemiological models are generally defined as mathematical and/or logical demonstrations of epidemiology of disease and the related process. Epidemiological disease models provide a way to eliminate these uncertainties by combining knowledge from field and experimental studies and experts' views on the dynamics of infection and disease control (2).

Modelling might be useful when experimental or field studies are impossible or not practical (3) or in retrospective analyzing of previous epidemics in order to search alternative control strategies (4).

The first known epidemiological mathematical model was formulated and solved by Danial Bernoulli in 1760 (5). Even though mathematical modelling in epidemiology has a long history, dynamic system approaches started to be popular in 1920 s. In the early $20^{\text {th }}$ century, partial models were founded. From mid $20^{\text {th }}$ century, mathematical epidemiology studies increased incrementally (6). Since then, conceptional and technical developments have been spotted in models (7). Modelling in infectious diseases has recently been an important field due to the increase of avian influenza, swine influenza and many other diseases. Infectious diseases appear repeatedly and cause threat, and this situation increases interest everyday on the necessity of new and realistic statistical models about dynamics of diseases to understand spreading of infectious diseases $(7,8)$.

Models are used so as to predict the progress of epidemics and search the effect of model parameters. Change of model parameters might also change vaccination, treatment and other precautions (7). Mathematical models play a vital role in examining, explaining and predicting disease contamination dynamics and models of globally important diseases also play that role in controlling and developing public health strategies as a precaution (9).

In this study, the effect of mathematical modelling in the field of animal health on preventing infectious diseases from developing and spreading, evaluating treatment options and developing vaccination, control and protection programs was searched. In this sense, various modelling methods used in management of animal diseases were classified and necessary steps to create a model were explained.

\section{What is mathematical modelling?}

Infectious disease models are defined as the differential 
equation stipulating the dynamic contamination cycle including the interaction between the infected and susceptible host (9). Models are developed to understand the effect of external effects on outcomes by representation of interactions and to provide ideas about compounds and actions of the system (1).

Mathematical modelling enables us to explain the macroscopic behavior of the disease microscopically (10), to make a relation between behaviors at different scales and realizing the unknown out of a known situation (11, 12), to make use of our updated knowledge about the state and progress of an epidemic, to predict future and most importantly, to measure the uncertainties in these predictions (13).

The concept of modelling in infectious diseases is generally explained as models of infectious disease contamination, partial models or dynamic systems. A mathematical model uses a mathematical language to provide a better and more certain explanation of the system $(11,12)$.

While formulating a model for a special problem, there are three important points; accuracy, transparency and flexibility. Accuracy means to increase data and make a reliable prediction for future dynamics (12). Accuracy is usually developed by increasing model details. In order to obtain reliable results from data analysis, there should be a certain relation between the models obtained from experiences or field observations and the data. Host and pathogen heterogeneity is highly important. Another issue which is also as important is the frequency and type of transmission between infected and susceptible hosts $(12,14)$.

Transparency occurs by understanding how various compounds affect dynamics. Transparency is generally obtained by consecutive adding or removing of the compounds and building new things via simple models. When model compounds increase, it is more difficult to detect roles of compounds and interpret their interaction with the whole. For this reason, transparency is opposite accuracy most of the time (12).

Flexibility measures convenience by models that are adaptable to new situations. Flexibility is necessary to evaluate model control policies or predict future disease levels in constantly changing environments. As a result, accuracy of any model is always limited (12).

\section{Features of a good model}

Epidemiological models cannot respond questions at times; however, model operators can find answers to their questions with mathematical model by asking an interesting question and making the right combination (10). Selecting the model depends on the utility and quality of data, epidemiology of the disease to be modeled and experience of the operator, and detecting how complex a model should be is a kind of both science and art as well (15). It is clear that no model is perfect and no model can predict the result of infection period correctly. All models are simple forms of complex systems. The most suitable model type depends on the kind of the studied subjects. For example, deterministic models based on average and expected value parameters might be useful to understand simple infection dynamics. They have a limited usage as a prediction tool. However, when epidemiological information and quality data is accessible, more detailed models can be developed $(16,17)$.

In order for the model to be comprehensive, building a model should be started with asking specific questions. Selecting the model depends on how much the epidemiology of a disease is understood and on the amount and accessibility of data. Additional data might increase complexity. On the other hand, the ignored factors might be important for a model. The critical step during development of a model is the confirmation and approval periods. Confirmation should be within the frame of formulas and logical computer codes of model. Approval makes the model realistic. The meaning of all these is that assumptions lying under the model are true and the model is realistic $(2,16)$.

\section{Using models in management of animal diseases}

Recently, attempts have been made to develop realistic mathematical methods for infectious diseases contamination dynamics. Models provide us with idea production frames to conceptualize certain systems. Modelling can be used to compare different diseases within the same population, the same disease within different populations or the same diseases at different times (10). Besides, in animal diseases, using host and environmental factors together with interaction of parameters could be useful to understand the result of the disease. Therefore, models might create a budget friendly and logical basis for studies to evaluate effects and to test responds to interventions $(2,8)$.

Evaluating the possible results of epidemics and testing various control options provides an advantage on decreasing spread of diseases. However, evaluating alternative approaches to control infectious diseases is not easy and it requires thinking on some issues. These thoughts should provide answers to various subjects such as resources, commercial and economical effects, access to available technology (vaccines or diagnose tools), concerns of consumers and reflections on public health. Diseases create a great deal of concern for countries exporting animal or animal products especially. Occupational health and safety is also taken into account in zoonotic diseases. As a result, selecting control precautions for large scale applications is usually considered among necessities. Modelling is an important tool for these kinds of evaluations. Epidemiological and economical models used to control diseases are quite common (2).

\section{Steps followed during modelling}

The planned modelling activity is performed in two steps as creating the model initially and later controlling its accuracy and a modelling process widely contains activities in Figure 1.

\subsection{Defining systems and targets for modelling}

This is the most basic stage. It includes selecting suitable data to determine aims clearly, to define the system to be modeled and to monitor the action of the model (18). 


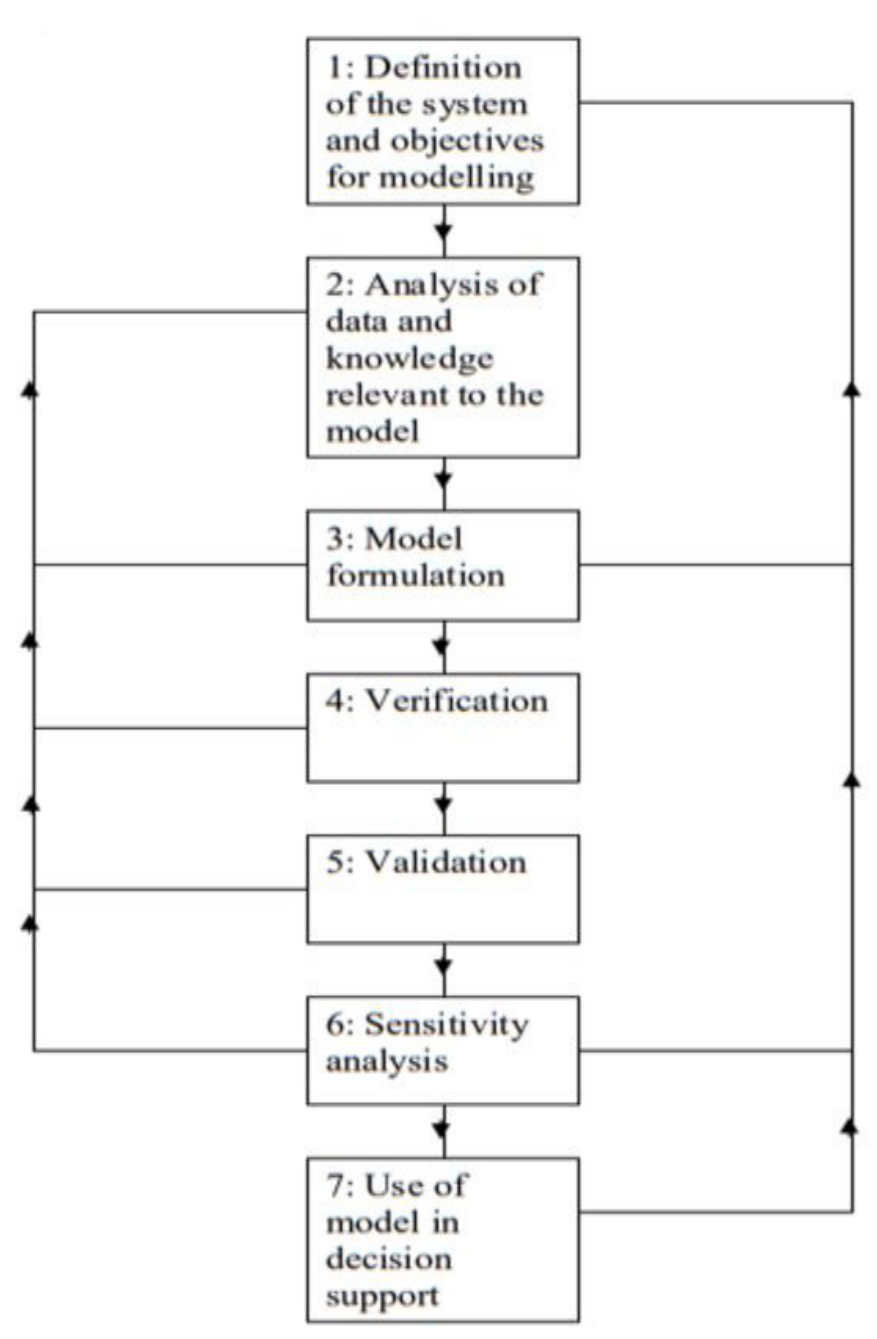

Figure 1. Stages in model building (19).

Target of modelling must have a clear statement. The specific model is expected to solve problems and support decisions with the detailed desired information and the defined model $(11,19)$.

\subsection{Information and data analysis concerning the model}

In model developing, this step is similar to danger defining stage of risk analysis and aims to develop a list of factors that might affect the accuracy and certainty of selected model data (1). These factors and mutual relations are converted to mathematical (logical) model structure on which quantitative can be added. This stage might contain analysis of field and experimental data, literature examinations and expert opinions. Cooperation between model operators and experts (vets, virologists, microbiologists, biostatisticians etc.) is highly crucial in developing a valid model conceptionally. Close cooperation between model creators and specialization experts is very important at this stage $(11,19)$.

\subsection{Model Formulation}

A conceptional model is an oral or graphical representation of the examined system (20). The selected modelling method should be converted to a document explaining model assumptions and parameter predictions (18).
While selecting a certain modelling approach, model operators should consider the study population and how to represent the progress of infection in individuals, time, environmental factors and contamination of infection. These decisions will also affect the methods used to analyze the obtained results. The balance between model complexity and data requirements is quite important because availability and quality of data is a limiting factor in developing epidemiological models (1).

In this stage, features of the related system, events happening here and ongoing procedures are defined and aims of the model are stated. Model formulation consists of steps that need to be carried out in computers. Considering the existing assumptions, data analysis is performed and a suitable mathematical model is created by calculations $(11,19)$.

\subsection{Confirmation}

Confirmation is the step where model operators control whether theories and assumptions in the model are suitable to the planned usage of the model and whether their action plans are carried out or not (19). One of the confirmation techniques is to ask for comments on relevance of the design from experts (20).

\subsection{Validity}

Validity of the model is related with controlling whether models are correct for real life. Objective and universal tests unfortunately do not exist for model validity. Yet, some common themes on models in literature can be instructive (11, 19).

Validity of a model could be evaluated by results of other models (21), internal behavior of the model and comparing the results with results of the real system (20).

Valid models are biologically logical. Disease modelling should not be carried out separately from other epidemiology branches and reflecting the truth is an important image of modelling. The model must take proper actions biologically and mathematically. For example, it must be susceptible to suitable variables. Explaining the results of the model must be possible according to updated biological information. One of the benefits of modelling is that it enables us to better understand the basic biology lying under the modeled system $(11,19)$.

\subsection{Analysis of susceptibility}

When data validity is limited, the importance of uncertain parameters can be evaluated by carrying out studies on analysis of susceptibility (22). Input parameters of a model can systematically be changed by searching how the uncertainty and variation in parameter predictions affects the outputs (23).

In this stage, availability of the model is tested, the model is confirmed and analysis of susceptibility is performed (19). The analysis of susceptibility includes the evaluation of effects of changes in input values on model outputs (22).

Analysis of susceptibility has two aims: 
To control the susceptibility of the model against low quality data

To control the susceptibility of the model against the known changes in system parameters.

Analysis of susceptibility is used for uncertainties in parameters especially when there is a lack of quality data. Uncertain parameter values are diversified to detect the effect of the model on the result. The susceptibility of the model against parameter values is evaluated. Even though a model with little reliable data is susceptible to parameters, its usage might provide negative outcomes for the decision. On the other hand, if the changed parameter has little effect on the result, the uncertainty about the value of this parameter does not decrease model value in decision support model. The fact that there are parameters known to change and be susceptible all in all provides benefits about direct rule making for model management because if these are parameters that could be affected by disease control activities, they become 'critical control points' to be monitored and controlled during an epidemic. Analysis of susceptibility of a model which is susceptible to a parameter proves that this susceptibility is higher in real life. This situation shows the necessity to reformulate and confirm the model. $(11,19)$

\subsection{Usage of the model as decision support}

During the final stage, results of the model are combined with other information by decision maker. Later model is included in usage. However, new data from other resources and unexpected results of the model might cause a swift change in the model and even require redefinitions of the targets for modelling $(11,19)$.

\section{Classification of models used in the field of animal health}

There has not been a reconciliated classification system for models so far. Models of diseases can be classified within various categories depending on variable treatments, time, environment and population structure. There are different approaches ranging from simple deterministic models to complex stochastic simulations $(2,16,19)$.

\subsubsection{Classification of models according to their} structures

\subsubsection{Static and Dynamic Models}

Static models represent the condition of a system while tackling its condition at a certain time. Time factor is also important in dynamic models; however, it is used to understand the condition of a system within a certain interval of time. Static model claims that variables do not change in time while dynamic model claims the opposite. For example, in static modelling, as vaccine comprehensiveness increases, incidence of diseases is expected to decrease. However, following vaccination, it is known that incidence decreases, then increases, and later settles on a certain level and population immunity occurs. Therefore, dynamic modelling is a more convenient option $(11,14)$.

\subsubsection{Deterministic and Stochastic Models}

Deterministic models are the systems that do not contain any elements of randomness, uncertainty and chance in detecting future conditions of the system. They merely explain the average tendency of a period. By assuming that relations between the elements forming the existing system and the related data are certainly known, modelling is performed, possibilities are not taken into account and rough and average results are obtained. For a well modeled deterministic system, the system always provides the same result under the same circumstances and the same starting conditions $(2,8,14,24)$.

It is inevitable for epidemic models to avoid environmental variables. In order to reflect the positive realistic effect of these variables that are ignored in deterministic models, many stochastic models have been developed and used in epidemic populations. Stochastic models are the systems including randomness. This randomness might exist within system parameters, dynamics and inputs. Therefore, the output of the system has a similar randomness. For these kinds of systems, same results might not be obtained even when the same experiment is performed under the same conditions. However, it can be calculated at which intervals or distributions the results should be and which result is possible to appear. In stochastic models, instead of detecting the future position of the system, the point is to predict the system and make it probable. One or more system variables, risks and uncertainties looking like incidental in stochastic models are taken into account and simulations are created by regarding the chance factor $(11,8$, $25,25)$.

Deterministic model is regarded to have certainly known, unchanged and uncontrolled data while stochastic model is considered to have uncontrolled data with uncertainties and variations (27).

A deterministic model always provides the same result for steady starting values while a stochastic model produces many different outputs depending on the real values of random variables (28).

Stochastic models are more realistic compared to deterministic models (11). The reason for this is that the effect of changing events (24) and the variability of the nature is reflected onto the final result and a range of possible results are obtained (26).

\subsubsection{Optimization and Simulation Models}

Optimization models are composed of mathematical statements reflecting the function and features of the system and including the interactions within the system and others around. Optimization models consider a set of criteria and limitations and aim a certain purpose to happen in an optimum way. Optimization technology is used for accelerating decision periods, increasing decision quality and effective, accurate and real time solving of real life problems $(11,14)$.

Simulation models reveal the effect of various fictions on the system by imitating the related system (11). 


\subsubsection{Classification of models according to their aims}

\subsubsection{Statistical/Epidemiological Models}

Epidemiological model is usually defined as the mathematical orlogicaldemonstration of disease contamination epidemiology and related operations. Models examine the causes affecting occurrence, condition, contamination, size and direction of diseases. Quantitative models provide contamination dynamics of animal diseases among animal groups in a certain time and environment. For that reason, an epidemiological model helps to evaluate the effects of potential control precautions and to predict the future size of an epidemic with special control precautions. However, epidemiological models for animal disease management are more widely defined as containing a set of statistical/mathematical models. The greatest advantage of using models in animal health is that they are able to analyze behaviors of real epidemics beforehand. Presumptive scenarios can be improved to develop different strategies in different situations $(2,11,29)$.

Modelling offers the following contributions to disease control:

- Retrospective analysis of previous epidemics and evaluation of different control strategies

- Discovery of different strategies in presumptive epidemics (probability plans)

- Discovery of resource requirements for different strategies in presumptive epidemics (resource planning)

- Detecting primary fields in risk evaluations and preparation for risks

- Evaluation of various activities of observation strategies

- $\quad$ Supportive economical effect studies

- Communication between realistic scenarios and epidemiology and disease control

- $\quad$ Providing support during epidemics by analysis and hypothesis

Models can be used retrospectively and prospectively. Retrospective ones use epidemiological data and mathematical equations to comment on these data. Prospective ones either use updated data to predict the progress of an epidemic or are performed by a set of possible epidemiological scenarios instead of focusing on a certain case. These types of models are generally used for probability plans (2).

\subsubsection{Economical Models}

Economical models reveal the economical effects of the disease and offer a wide range of solution suggestions. Modelling techniques used in the field of animal health economy help to reveal economical effects on producers, marketing channels and governments in epidemic scenarios, developing control/eradication strategies and evaluating the existing and possible conditions about animal diseases and they provide important technical and economical contributions (11).

\subsubsection{Classification of models according to their} contents

Contamination models used in infectious diseases classify members of population as susceptible, sick and recovered. Only two or three sections are used in simple contamination models while more complex conditions can be departmentalized further $(24,30)$.

'S' stands for susceptible population, ' $\mathrm{T}$ ' infectious population, ' $R$ ' recovered population after an infection, ' $E$ ' population in latent period and ' $\mathrm{M}$ ' passive immune newborn population.

There are three important rules in modelling:

- Total population is mixed and each individual is susceptible to disease.

- The disease is spread by contact of disease susceptible individuals with sick individuals.

- $\quad$ Each sick and recovered individual is regarded as immune against the disease.

In this case, each susceptible individual becomes infectious suddenly and then recovers owing to temporary immunity acquired later $(7,24,30)$.

\subsubsection{Models at simple level}

\subsection{SIR Modelling}

SIR is one of the basic models. It is a basic simple level modelling method $(7,24,30)$. SIR model is used if individuals acquire permanent immunity at the end of the disease (10).

SIR modelling method could be set up for epidemics caused by a certain pathogen with a great deal of data. In order to set this model, information on incidence and immunity is needed (31). When data on the epidemic is little or non-realistic, SIR model has a tendency of being ineffective $(13,31)$.

This modelling method is usually used for acute (7) and viral (10) infections such as influenza, rabies, measles and chicken pox. SIR modelling is the best mathematical modelling used for acute infections appearing after immunity (7).

\subsection{Death from infections and SI Modelling}

In SIR model which assumes the infection as benign, recovering and disease occurs in one period of the lifelong immunized disease. This is a convenient modelling for extensive infections such as chicken pox and influenza. However, for diseases with vital death risk such as malaria, measles, chin cough and SARS, SI modelling is used by adding ' Infection Based Death rate' to the equation in order to find death possibility especially. Yet, it could be complex to comment on this biologically or predict from data. Instead, considering the probability of (I) individual died from infection in infective group might be a better preference. Birth and natural death 
could be ignored in these types of fast spreading diseases (12).

\subsection{Without Immunity: SIS Model}

SI and SIR models both tackle dynamics of acute infections that kill or provide lifelong immunity when recovery happens. Many contaminant diseases such as rotaviruses, sexually transmitted infections and most bacterial infections, nevertheless, do not provide long term immunity. Individuals might get infected many times without acquiring immunity in these diseases all life long (12). SIS model is used for diseases for which the infection does not provide immunity since there happens an instant rotation towards the susceptible group when recovery from the infection occurs (10). Even though new born susceptibles are ignored, the disease might continue due to the fact that recovered patients participate in the susceptible group (12). This method is mostly used for bacterial diseases (bacteria based meningitis and Streptococcus based pharyngitis) and protozoa based diseases (malaria) (10).

\subsection{Latent Period: SEIR Model}

The individual should be in contact with the factor sufficiently before the infection begins in many diseases such as tuberculosis and leprosy. SEIR modelling is a method obtained by making developments on SIR and SIS models to consider latent period. In this method, the individual in latent period is regarded within an extra group $(12,32)$.

The time period that appears during the interval when the pathogen replicates quickly in the host is the period when immunity system partly cannot struggle. During this period, amount of pathogens is quite low for active contamination to other susceptibles, however, pathogens exist. Therefore, the host cannot be regarded as a member of susceptible, contaminant or recovered categories. For this reason, a new category is to be defined for infected but non-contaminative individuals. The individual is susceptible at the beginning, but then is exposed to the factor (within the latent period), gets sick and recovers by permanent immunity $(12,32)$.

Hosts regarded as exposed to diseases and latent period group variable are indicated with ' $E$ ' and a differential equation is produced in SEIR model $(12,30)$. Dynamic features of SEIR model are similar to those of SIR model qualitatively (12).

\subsection{SIRS Modelling}

SIRS modelling is used when individuals acquire temporary immunity at the end of the disease. Individuals with temporary immunity are not included in the susceptible group until they lose their immunity and are counted in the susceptible group after they lose their immunity. The model turns into SI model when individuals do not recover (10).

\subsection{Continuous and Discontinuous Time Models}

Continuous time models are effective in terms of calculations, however, might not represent irregularly happening events in a realistic way (1).

Discontinuous models were first developed by Reed and Frost in 1928. Discontinuous time models divide time into equal units and model population is updated incrementally at each time interval. Selecting an appropriate time unit mostly depends on infection dynamics and quality of data (1).

\subsubsection{Advanced Modelling}

\subsection{SEIS Modelling}

Some disease factors undergo a latent period for a while before they cause disease in the host. In these diseases, individuals first get into a latent period and later the infection begins (30).

In some studies, latent period is not taken into account but it is important. Because some diseases like tuberculosis might result in death when they are not treated. SEIS modelling occurs when latent period is added into modelling. Recovered individuals acquire susceptibility and get back to ' $\mathrm{S}$ ' group in this model. In this modelling, 'S' stands for susceptible group, 'E' latent group and 'I' infected group $(30,33)$.

\subsection{MSIR Modelling}

The newborn might have a passive immunity against some diseases thanks to maternal antibodies they take from mothers. For this reason, a separate group stated as ' $\mathrm{M}$ ' is added into modelling for passive immune newborn (Figure 2). When maternal antibodies move away from the body, individuals are included in 'S', that is susceptible group $(34,35)$.

\section{Birth with passive immunity}

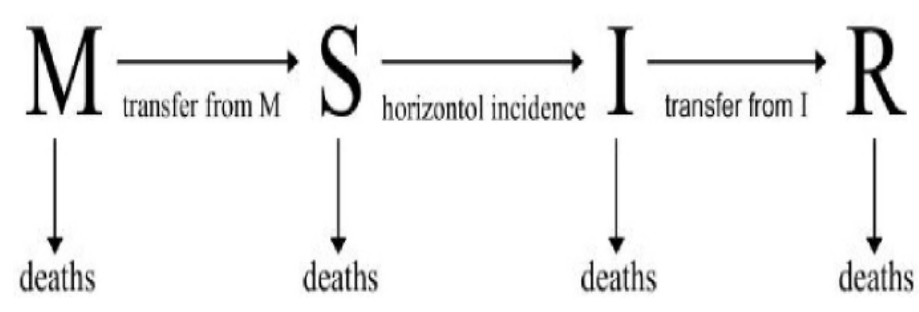

Figure 2. General Transfer Diagram of MSIR Model (M: passive immune group, S: susceptible group, I: infective grup, R: immune group)

\subsection{MSEIR Modelling}

In this modelling method, 'M' group for passive immune individuals thanks to maternal antibodies from mothers and ' $E$ ' group for those in latent period are both included in modelling (Figure 3) (36).

If the mother is infected by a factor and the existing IgGs are transmitted to the baby, the baby is born as passive immune against the disease. After maternal antibodies move away from the body, the individual is transferred to susceptible group (S group). Babies whose mothers are not infected are 


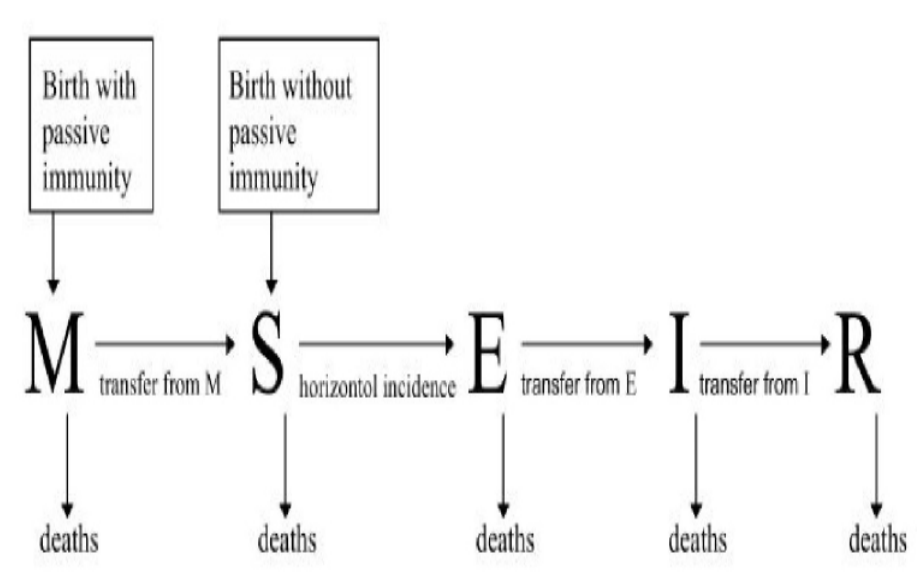

Figure 3. The general transfer diagram for the MSEIR model (M: passive immune group, S: susceptible group, E: latent group, I: infective group, R: immune group) (36).

born without immunity and are directly included in S group. When susceptible individuals contact with the factor, they undergo latent period until they display disease symptoms and they are regarded in ' $E$ ' group. When latent period is over, the individual is in the infectious group and they now have the ability to contaminate the disease. When infectious period is over, the individual is in the immune group ( $\mathrm{R}$ group). Which groups should be included in the model depends on the character of the disease and the aim of modelling. Passive immune group $\mathrm{M}$ and latent period group $\mathrm{E}$ are mostly skipped, because it is not very important for susceptible-infective interaction (36).

\subsection{MSEIRS Modelling}

MSEIRS modelling is similar to MSEIR modelling; however, the immunity acquired following the infection is temporary. That is to say since the immunity in R group is temporary, individuals acquire their susceptibility again and get into the susceptible group when temporary immunity is over (Figure 4) (25).

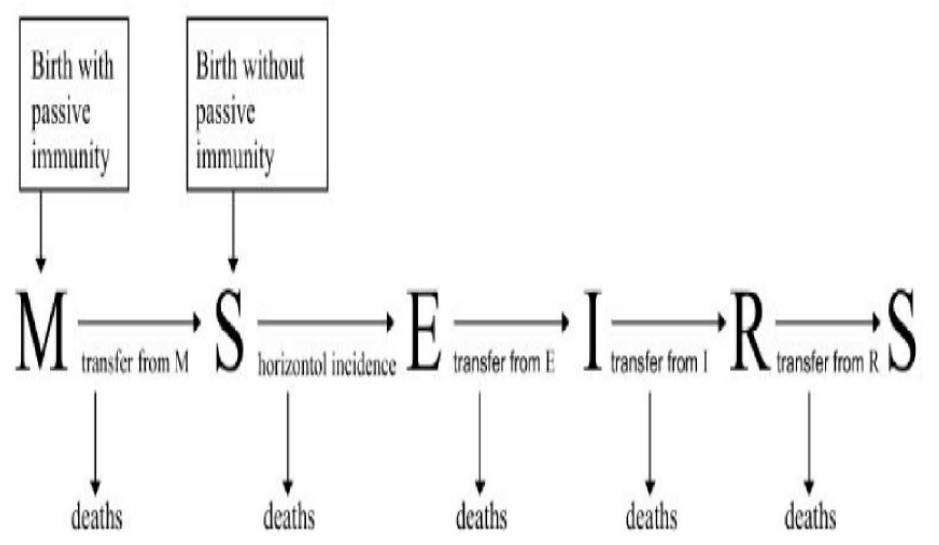

Figure 4. The general transfer diagram for the MSEIRS model (M: passive immune group, S: susceptible group, E: latent group; I: infective group, R: immune group) (25)

Mathematical modelling has been defined and information has been provided on steps followed during model preparations and why it is used. Modelling methods used in the field of animal health have been classified according to their structures, aims and contents. Some methods used in animal disease management have been revised and different modelling approaches have been shortly explained.

In this study, instructions are provided for points to consider while designing a model and critical evaluation of models. Besides, process of future possible epidemics can be predicted by the help of data from previous epidemics and epidemics can be prevented by the help of control programs. Models are used to predict process of epidemics and to search the effect of model parameters.

Using modelling in animal health is important in terms of vaccination, treatment, control precautions and developing public health strategies. However, using modelling in veterinarian field and reflections over the field in Turkey is very rare. On this topic, veterinarians should be raised and specialized; they should work in harmony with their field and other model operators (virologists, microbiologists and biostatisticians etc.) As a result of this, it is believed that both animal health will be protected and important contributions to the economy of the country will be offered.

\section{CONFLICT of INTEREST}

The authors report no conflicts of interest. The authors alone are responsible for the content and writing of the paper.

\section{REFERENCES}

1. Garner MG, Hamilton SA. Principles of epidemiological modelling. Rev Sci Tech Off Int Epiz. 2011;30 (2): 407-416.

2. Dubé C, Garner G, Stevenson M, Sanson R, Estrada C, Willeberg P. The use of epidemiological models for the management of animal diseases, Conf. OIE. 2007a.p. 13- 23.

3. Garner MG, Beckett SD. Modelling the spread of footand-mouth disease in Australia. Aust Vet J. 2005;83(12): 25866.

4. Mangen M, Jalvingh A, Nielen M, Mourits M, Dijkhuizen AA, Dijkhuizen A. Spatial and stochastic simulation to compare two emergency-vaccination strategies with a marker vaccine in the 1997/1998 Dutch classical swine fever epidemic. Prev Vet Med. 2001;48, 177-200.

5. Zhou Y. Stability of periodic solutions for an SIS model with pulse vaccination, Mathematical and Computer Modelling. 2003;38: 299-308.

6. Braur F. Basical ideal of mathematical epidemiology. In: Castillo-Chavez C, Blower S, editors. Mathematical Approaches for Emerging and Reemerging Infectious Diseases. SpringerVerlag, New York, 2002. p. 31-65.

7. Gran JM. Infectious disease modelling and causal inference MSc, University of Oslo, Oslo, Norway. 2010.

8. Yang Q, Jiang D, Shi N, Ji C. The ergodicity and extinction of stochastically perturbed SIR and SEIR epidemic models with saturated incidence. J Math Anal Appl. 2012;388 (1): 248271. 
9. Wu J, Dhingra R, Gambhir M, Remais JV. Sensitivity analysis of infectious disease models: methods, advances and their application. J R Soc Interface. 2013;10: 20121018. http:/ / dx.doi.org/10.1098/rsif.2012.1018

10. Hethcote HW. Three basic epidemiological models. http: / / www.m tholyoke.edu/ ahoyerle/math333/ ThreeBasicModels.pdf . Accessed: 12.12.2019.

11. Can MF. Hayvan sağlığı ekonomisi alanında kullanılan modelleme teknikleri ve çeşitli modelleme çalışmaları. Vet Hekim Der Derg. 2009;80(3):7-12.

12. Kelling MJ, Rohani P. Modeling infectious diseases in humans\&animals, Clinical Infectious Diseases. 2008;47(6):864865.

13. Kelling MJ, Danon L. Mathematical modelling of infectious disease. British Medical Bulletin. 2009;92: 33-42.

14. Anon2. Optimizasyon modelleri ve çözüm metodlar1. http:/ / home.ku.edu.tr/ mturkay/indr501/Optimizasyon. pdf Accessed: 11.01.2020

15. Eddymurphy UA. Discrete modeling and analysis of contact networks in epidemic models, Master's Thesis, Lappeenranta University of Technology, Lappeenranta, Finland. 2018.

16. Garner MG, Dubé C, Stevenson MA, Sanson RL, Estrada C, Griffin J. Evaluating alternative approaches to managing animal disease outbreaks - The role of modelling in policy formulation. Vet Ital. 2007;43 (2): 285-298.

17. Green LE, Medley GF. Mathematical modelling of the foot and mouth disease epidemic of 2001: strengths and weaknesses. Res Vet Sci. 2002;73(3): 201-5.

18. Law AM. How to build valid and credible simulation models. https://ieeexplore.ieee.org/abstract/ document/1574236. Accessed: 09.01.2020.

19. Taylor N. Review of the use of models in informing disease control policy development and adjustment. https:// pdfs.semanticscholar.org/c8dc/8214ec760f8cd73f164e83aea4 03a98e54bb.pdf. Accessed: 17.01.2020.

20. Sargent R. Verification and validation of simulation models. https://www.informs-sim.org/wsc11papers/016.pdf. Accessed: 12.01.2019.

21. Dubé C, Stevenson MA, Garner MG, Sanson RL, Corso BA, Harvey N, Griffin J, Wilesmith JW, Estrada C. A comparison of predictions made by three simulation models of foot-and-mouth disease. NZ Vet J. 2007 b;55 (6), 280-288.

22. Frey HC, Patil R. Identification and review of sensitivity analysis methods. Risk Analysis. 2002;22 (3), 553-577.

23. Chapagain P, van Kessel J, Karns J, Wolfgang D, Hovingh E, Nelen K, Schukken Y, Grohn Y. A mathematical model of the dynamics of Salmonella Cerro infection in a US dairy herd. Epidemiol Infect. 2008;136, 236-272.
24. Gandolfi A. Percolation methods for SEIR epidemics on graphs. In: Rao VSH, Ravi D,editors. Dynamic Models of Infectious Diseases. 2013.p.31-58.

25. Anon1. Deterministik ve stokastik modeller. http:// ozgur aktekin.blogspot.com.tr/2015/09/deterministik-vsstokastik-mode ller.html Accessed: 09.01.2020

26. Ergönül Ö. Enfeksiyon hastalıkları epidemiyolojisi, Hastane enfeksiyonlar1: korunma ve kontrol sempozyum dizisi. 2008;80: 30-41.

27. Gürsoy ŞT, Öcek ZA. Bağışıklamanın ekonomik analizi, İnfeksiyon Dergisi. 2007;21 (4): 217-223

28. Choisy M, Guégan JF, Rohani P. Mathematical modeling of infectious diseases Dynamics. In: Michel T, editor. Encyclopedia of Infectious Diseases: Modern Methodologie, Wiley \& Sons, Inc., Hoboken, New Jersey, USA. 2007.p.382383.

29. Becker NJ. Using data to inform model choice, In: Chow SC, Jones B, Liu J, Peace KE, Turnbull BW (ed) Modeling to inform infectious disease control, CRC Press Taylor \& Francis Group. 2015. p 169.

30. Yang B. Stochastic dynamics of an SEIS epidemic model, Advances in difference equations. 2016;226. doi: 10.1186/s13662-016-0914-3.

31. Jenkins D. An examination of mathematical models for infectious disease, Honors research projects. 2015; projects 194.https://ideaexchange.uakron.edu/cgi/viewcontent. cgi?article $=1195 \&$ context $=$ honors_research_projects.

32. Li MY, Muldowney JS. Global stability for the SEIR model in epidemiology. Mathematical Biosciences. 1995;125: 155-164.

33. Hui J, Zhu DM. Dynamics of stochastic SEIS epidemic model with varying population size, Int J Bifurcation Chaos. 2007;17 (5): 1513- 1529.

34. Chapman JD, Chappell MJ, Evans ND. The use of a formal sensitivity analysis on epidemic models with immune protection from maternally acquired antibodies. Computer Methods and Programs in Biomedicine. 2011;104: 37- 49.

35. Bichara D, Iggidr A, Sallet G. Global analysis of multistrains SIS, SIR and MSIR epidemic models. J Appl Math Comput. 2013; 44(1-2):273-292.

36. Hethcote HW. The mathematics of infectious diseases, SIAM Review. 2000;42 (4): 599-653. 